\title{
The minimal important difference for the St George's Respiratory Questionnaire in patients with severe COPD
}

\author{
Jorrit B.A. Welling, Jorine E. Hartman, Nick H.T. Ten Hacken, Karin Klooster
} and Dirk-Jan Slebos

Affiliation: Dept of Pulmonary Diseases, and Groningen Research Institute for Asthma and COPD (GRIAC), University of Groningen, University Medical Center Groningen, Groningen, The Netherlands.

Correspondence: Dirk-Jan Slebos, Dept of Pulmonary Diseases, and Groningen Research Institute for Asthma and COPD (GRIAC), University of Groningen, University Medical Center Groningen, PO Box 30001, 9700 RB Groningen, The Netherlands. E-mail: d.j.slebosđumcg.nl

ABSTRACT The St George's Respiratory Questionnaire (SGRQ) is a validated, commonly used questionnaire for measuring quality of life in patients with chronic obstructive pulmonary disease (COPD). The current established minimal important difference (MID) for SGRQ scores in an average COPD population is -4 units. However, for patients with severe COPD, the MID has not been thoroughly validated. We re-determined the SGRQ MID for this patient group.

115 severe COPD patients (forced expiratory volume in $1 \mathrm{~s}$ (FEV1) $26 \pm 9 \%$ of predicted, SGRQ score 62 \pm 11 units; mean \pm SD, ) who participated in seven different bronchoscopic lung volume reduction clinical trials were included in the analysis. Anchor- and distribution-based methods were used to define the MID for SGRQ scores. FEV1, 6-min walk distance and residual volume were used as anchors.

Combining both anchor- and distribution-based methods, we identified a SGRQ MID of -8.3 units at 1 month and -7.1 units at 6 months.

This study proposes an alternative SGRQ MID for patients with severe COPD of -8.3 units at 1 month and -7.1 units at 6 months follow-up after intervention. Our new MID estimates could be applied for both interpreting SGRQ outcomes as well as sample size determination in future clinical trials investigating interventions in severe COPD patients.

@ERSpublications

In patients with severe COPD the redefined MID for SGRQ is -8.3 units at 1 month and -7.1 units at 6 months http://ow.ly/RSYO0 


\section{Introduction}

The St George's Respiratory Questionnaire (SGRQ) is a widely used, self-reported, quality of life assessment method to evaluate obstructive airways disease and especially chronic obstructive pulmonary disease (COPD) [1]. When evaluating different treatment options for COPD it is relevant to investigate whether a statistically significant improvement is also clinically relevant. A method to describe this relationship is called the minimal important difference (MID) [2].

A decrease of 4 units, after a medical intervention, in the SGRQ score is generally accepted in the literature to be a valid threshold value of beneficial treatment [3]. This threshold, or MID, is broadly applied in the evaluation of COPD treatment for patients with a wide range of disease severity. However, patients with severe COPD have not been specifically included in previous MID calculations, knowing that their baseline SGRQ total scores are very high, making a 4-unit drop easy to achieve. In our own clinical experience, many patients with severe COPD improve $>10$ units after bronchoscopic lung volume reduction treatment [4-6]. Therefore, the established 4-unit MID for SGRQ scores might not be fully applicable to the severe COPD patient group. We hypothesised that the MID for SGRQ scores is higher in patients with severe COPD.

The objective of this study was to determine the MID of SGRQ scores in patients with severe COPD.

\section{Methods}

\section{Study design}

We performed a retrospective analysis on data from seven completed bronchoscopic lung volume reduction (BLVR) clinical trials conducted in one hospital in The Netherlands (University Medical Center Groningen). Patients were treated with either airway bypass stents (www.clinicaltrials.gov identifier NCT00391612) [7]), coils (NCT01220908 [4], NCT01328899 [8] and NCT01421082 [9]), valves (NCT01101958 [6]) or foam sealant (NCT01449292). Two trials (NCT01101958 [6] and NCT00391612 [7]) included a control group and in total 19 control patients were included in our analyses. All trials had prior approval from the local medical ethical committee and all patients provided informed consent before participating. Patients were included in the analysis when 1-and/or 6-month post-treatment follow-up of the SGRQ assessment was available for evaluation.

\section{MID calculation}

Both the anchor-based as well as the distribution-based methods for determining the MID were used. For the anchor-based method, where the change in SGRQ outcome is compared with an established MID [2], the anchors chosen were: the forced expiratory volume in $1 \mathrm{~s}$ (FEV1) (MID $100 \mathrm{~mL}$ [10]), 6-min walk distance (6MWD) (MID $26 \mathrm{~m} \mathrm{[11])} \mathrm{and} \mathrm{residual} \mathrm{volume} \mathrm{(RV)} \mathrm{(MID} 400 \mathrm{~mL}$ [12]).

Distribution-based methods compare the change in outcome measure with some measure of variability [2]. In this study, Cohen's effect size was used, which is a frequently used distribution-based method in studies establishing MIDs in pulmonary medicine [11-13] and is one of the distribution-based methods recommended in the literature $[2,14]$.

We calculated the final MID on the basis of both the anchor- and distribution-based methods. The combined MID was calculated using the average of the three anchor-based MIDs and one distribution-based MID, each counting for $25 \%$ weight.

\section{Measurements}

The SGRQ consists of three subscales and a total score. SGRQ scores range from 0 to 100 , with higher scores indicating worse quality of life. The total score summarises the impact of the disease on overall health status [15]. The SGRQ was completed at baseline, and at 1 and 6 months follow-up. Furthermore, spirometry and body plethysmography (MasterScreen ${ }^{\mathrm{TM}}$; VIASYS, Höchberg, Germany) were performed according to the American Thoracic Society/European Respiratory Society guidelines [16, 17], and a 6-min walk test was performed according to the American Thoracic Society guidelines [18].

\section{Data analysis}

The absolute and relative changes at 1 and 6 months follow-up compared with baseline were calculated. Pearson correlation coefficients (data were normally distributed) were calculated to test whether the univariate association between change in SGRQ score and change in anchor scores was sufficient to perform the anchor-based method. A linear regression analysis was performed with change in SGRQ score as dependent variable and change in anchor score as independent variable. Afterwards, we entered the anchor MID into the equation derived from the linear regression analysis. Subsequently, the MID could be calculated from the established equation. For the distribution-based method, a moderate (0.5) Cohen's effect size was calculated for the SGRQ score change from baseline to 1 and 6 months follow-up. p-values 
$<0.05$ were considered significant. Statistical analyses were performed using SPSS version 22 (IBM, New York, NY, USA).

\section{Results}

115 patients had a baseline SGRQ measurement in combination with either a 1- or 6-month follow-up SGRQ measurement and were included in the analyses. 110 patients were included in the 1-month follow-up analysis and 86 patients were included in the 6-month follow-up analysis (see figure 1 for study flowchart).

Patient characteristics at baseline and change scores after 1 and 6 months follow-up are shown in table 1. At baseline, FEV1 was $26 \pm 9 \%$ of predicted and the SGRQ total score was $62 \pm 11$ units (mean \pm SD).

An association of $r \geqslant 0.4(p<0.05)$ was found between the change in SGRQ score and change in all three anchors (figure 2).

The outcomes of the calculations for the anchor-based, distribution-based and combined methods at 1 and 6 months follow-up are shown in table 2. At 1 month follow-up, we calculated a SGRQ MID of each anchor: RV -8.7 (relative $-13.8 \%$ ), FEV1 $-9.2(-13.0 \%)$ and 6MWD $-8.5(-15.3 \%)$. At 6 months follow-up, the calculated SGRQ MIDs were: RV -6.4 (-10.2\%), FEV1 $-7.8(-11.5 \%)$ and 6MWD -6.3 $(-9.2 \%)$. With the distribution-based method, we calculated SGRQ MIDs at 1 month follow-up of -6.9 units (relative $-11.3 \%$ ) and at 6 months follow-up of -7.9 units (relative $-13.3 \%$ ). Our combined MID (average of the three anchor-based MIDs and one distribution-based MID) was -8.3 units (relative $-13.4 \%$ ) at 1 month and -7.1 units (relative $-11.1 \%$ ) at 6 months.

\section{Discussion}

To the best of our knowledge, we established for the first time a SGRQ MID specifically for patients with severe COPD. Our findings differ substantially from the SGRQ MID of -4 units that has been described more generally in COPD [3]. Our results showed that the MID for patients with severe COPD was -8.3 units at 1 month follow-up and -7.1 units at 6 months follow-up. The relative MID was $-13.4 \%$ at 1 month follow-up and $-11.1 \%$ at 6 months follow-up.

Our calculated SGRQ MID for patients with severe COPD significantly differed compared with the earlier established MID [3]. One explanation could be the chosen time points. In Jones et al. [3], it is not clear for which time point the SGRQ MID was determined. Whilst inconclusive, most of their methods to establish the MID were applied at the 12-month follow-up interval. In contrast, our MID estimations are

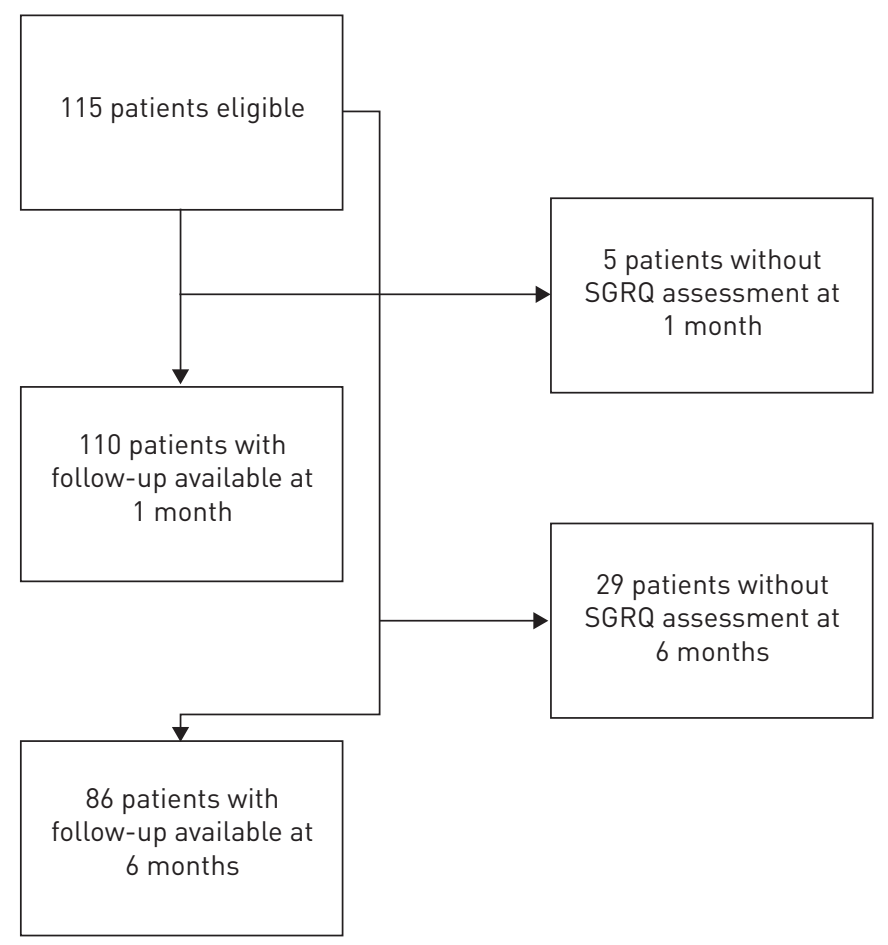

FIGURE 1 Patient flowchart. SGRQ: St George's Respiratory Questionnaire. 


\begin{tabular}{|c|c|c|c|c|}
\hline & Baseline & $\begin{array}{l}\text { Per cent predicted } \\
\text { at baseline }\end{array}$ & $\begin{array}{l}\Delta 1 \text { month from } \\
\text { baseline }\end{array}$ & $\begin{array}{c}\Delta 6 \text { months from } \\
\text { baseline }\end{array}$ \\
\hline Subjects $n$ & 115 & 115 & 110 & 86 \\
\hline Females/males & $73 / 42$ & NA & NA & NA \\
\hline Age years & $60 \pm 8.8$ & NA & NA & NA \\
\hline BMI $\mathrm{kg} \cdot \mathrm{m}^{-2}$ & $24 \pm 3.6$ & NA & $0.3 \pm 0.8$ & $0.5 \pm 1.1$ \\
\hline 6MWD m & $311 \pm 95$ & NA & $38.5 \pm 60.7$ & $38.2 \pm 68.5$ \\
\hline SGRQ total score units & $62 \pm 10.9$ & NA & $-9.8 \pm 13.8$ & $-7.5 \pm 15.8$ \\
\hline Pack-years & $36 \pm 17$ & NA & NA & NA \\
\hline RV L & $4.9 \pm 1.1$ & $241 \pm 46.7$ & $-0.5 \pm 0.6$ & $-0.5 \pm 0.6$ \\
\hline FEV 1 L & $0.7 \pm 0.3$ & $25.9 \pm 9.0$ & $0.1 \pm 0.2$ & $0.1 \pm 0.2$ \\
\hline TLC L & $7.7 \pm 1.4$ & $136 \pm 14.6$ & $-0.2 \pm 0.4$ & $-0.25 \pm 0.4$ \\
\hline RV/TLC \% & $64.6 \pm 7.7$ & $168 \pm 22.2$ & $-4.0 \pm 5.7$ & $-4.1 \pm 6.3$ \\
\hline
\end{tabular}

Data are presented as $\mathrm{n}$ or mean \pm SD. $\Delta$ : change between baseline and follow-up; BMI: body mass index; FEV1: forced expiratory volume in 1 s; 6MWD: 6-min walk distance; SGRQ: St George's Respiratory Questionnaire; RV: residual volume; TLC: total lung capacity; NA: not applicable.

established at the short-term, after 1 and 6 months follow-up. At the 6-month follow-up interval, the established MID was lower compared with the 1-month follow-up interval. It can be hypothesised that the diminished perceived effect of treatment results in lower decrease of SGRQ scores after 6 months follow-up. In the long-term, a MID could be further decreased due to the progressive nature of COPD. For example, although treatments may produce an initial improvement in SGRQ scores, scores may subsequently return to the baseline level or worse due to disease progression $[19,20]$. More research is needed to investigate whether the MID in severe COPD patients at 12 months follow-up differ from our findings at 1 and 6 months follow-up.

Our study population consisted of patients who were treated with BLVR and the question arises whether our newly established MID is only applicable to this treatment or to other treatment modalities for patients with severe COPD as well. There is no consensus on whether a MID is applicable to a specific treatment or a broad range of treatment modalities. JONEs et al. [21] state that MIDs were originally not developed in the context of specific treatments. They stated that the reference point (at least with anchor-based methods) is patient- or clinician-perceived benefit [21]. Troosters et al. [22], however, question whether MIDs based on anchor techniques can be used across different interventions as several processes may underlie the observed effects of interventions. For example, the expectations of patients may be different, yielding different effect sizes with different interventions [22]. Therefore, more research is needed regarding the specificity of MIDs for different treatments.

Several methods for estimating MIDs have been described in the literature, the most important being anchor-based and distribution-based methods [14]. The combination of multiple methods is generally recommended to increase reliability of outcome [14]. Therefore, we relied on both anchor-based and distribution-based methods. The anchor-based method requires that a reasonably strong linear relationship exists between the anchor and the variable of interest [23]. However, there is no consensus on how strong this relationship should be exactly. One review recommends statistically significant $(\mathrm{p}<0.05)$ correlation coefficients of $\geqslant 0.3$ as appreciable [14] and two studies in COPD patients performed the analyses when correlation coefficients were 0.3 or $0.5[11,24]$. In line with our previous study, we chose a correlation coefficient threshold $\geqslant 0.4$ [12]. Distribution-based methods are commonly considered inferior to anchor-based methods because they rely solely on statistical criteria and depend heavily on the characteristics of a particular study [23]. We agree with the general consensus that the distribution-based method should only be used to support estimates derived from anchor-based methods $[2,14]$. Therefore, we have placed less weight on the distribution-based method in calculating our combined MID.

The quality of the anchors can influence the outcomes when performing an anchor-based analysis. A good anchor, in our opinion, is present if it is derived from several studies with comparable patient populations, in our case severe COPD patients. The anchor should be determined using several methods. Finally, there should be an appreciable association between the outcome variable and anchor [23]. Here, we will evaluate the strengths and weaknesses of the three anchors we used for our analysis. The 6MWD MID estimate was determined in a study investigating the effect of lung volume reduction surgery in severe COPD patients [11]. Therefore, the patient population was quite comparable to ours, but a different intervention was 
performed. Another study, which investigated the MID for 6MWD, found a similar outcome for treatment with pulmonary rehabilitation [24]. This 6MWD anchor was moderately correlated with SGRQ scores in our study ( $\mathrm{r}=0.5$ ). The MID for RV was established in almost the same population as we used (93 patients
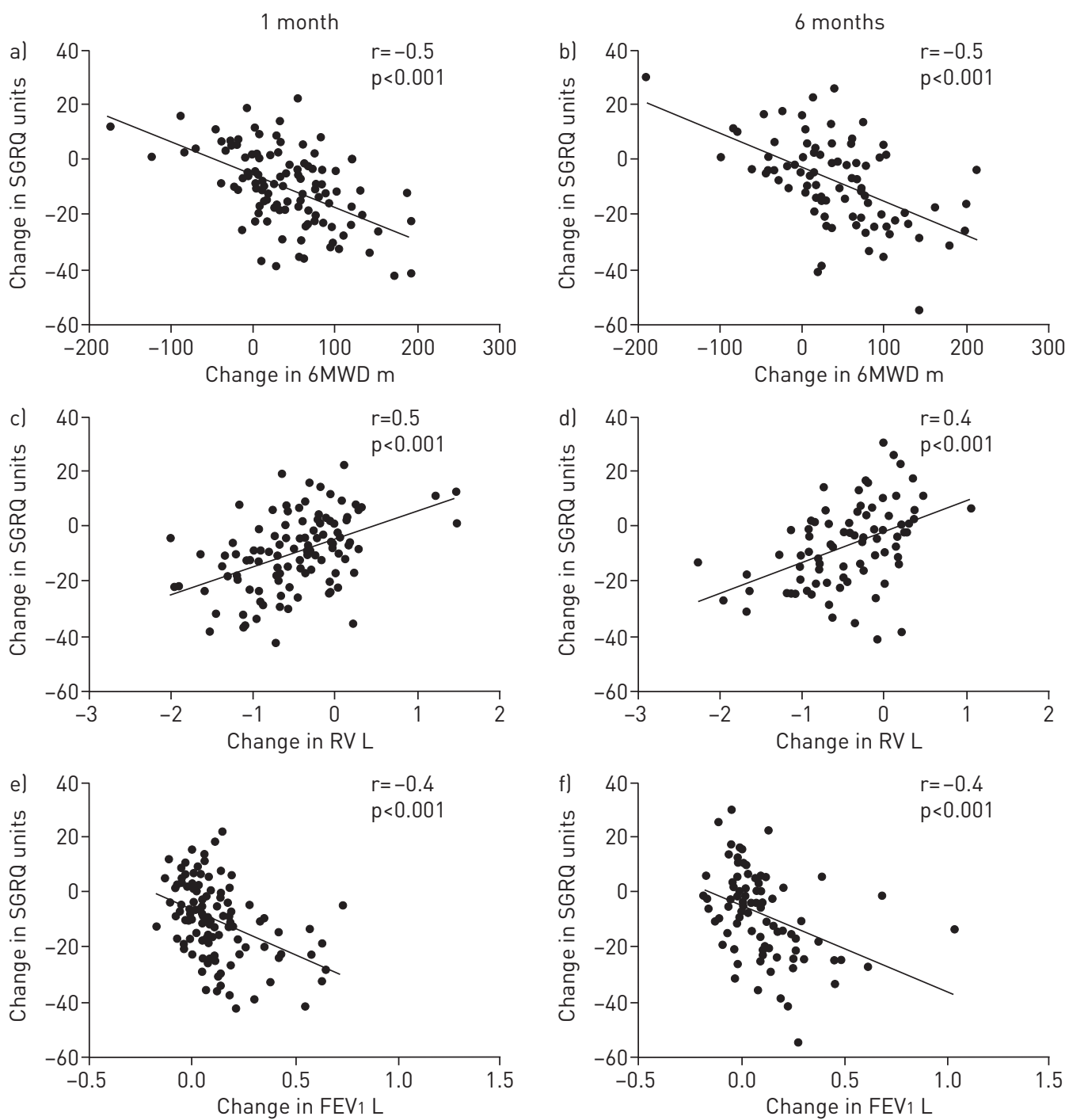

FIGURE 2 Scatterplots of 6-min walk distance (6MWD), residual volume (RV) and forced expiratory volume in $1 \mathrm{~s}$ (FEV1) versus St George's Respiratory Questionnaire (SGRQ) at a, c, e) 1 and b, d, f) 6 months follow-up.

TABLE 2 St George's Respiratory Questionnaire (SGRQ) minimal important differences (MIDs)

\begin{tabular}{lcccc} 
Methods & $\begin{array}{c}\text { Absolute SGRQ } \\
\text { MID 1 month (unit) }\end{array}$ & $\begin{array}{c}\text { Relative SGRQ } \\
\text { MID 1 month (\%) }\end{array}$ & $\begin{array}{c}\text { Absolute SGRQ MID } \\
\text { 6 months (unit) }\end{array}$ & $\begin{array}{c}\text { Relative SGRQ MID } \\
\text { 6 months (\%) }\end{array}$ \\
\hline Anchor-based & & & & \\
$\quad$ RV & -8.7 & -13.8 & -6.4 & -10.2 \\
FEV 1 & -9.2 & -13.0 & -7.8 & -11.5 \\
6MWD & -8.5 & -15.3 & -6.3 & -9.2 \\
Distribution-based & -6.9 & -11.3 & 7.9 & -13.3 \\
Combined & -8.3 & -13.4 & -7.1 & -11.1 \\
\hline
\end{tabular}

Data are presented as SGRQ units or percentage change compared with baseline. RV: residual volume; FEV1: forced expiratory volume in $1 \mathrm{~s}$; 6MWD: 6-min walk distance. 
overlap) and therefore is not the best anchor for establishing our MID [12]. The MID was determined in one single study and we feel it should be re-tested in more studies to increase the reliability. The MID for RV was moderately associated with SGRQ score $(r=0.4)$. A positive feature of the MID for FEV1 is that it was determined in several studies comprising general COPD populations [10]. A downside was that determining the MID of FEV1 was never the primary objective of these studies [10]. In our study, FEV 1 was moderately associated with SGRQ scores $(r=0.4)$. In contrast to the anchor-based MIDs, the distribution-based MIDs were found to be higher at 6 months follow-up than at 1 month follow-up. This can be explained by the fact that, inherent to their methodology, distribution-based methods depend highly on variance in the population. On 6 months follow-up, the variance of measurements in the population is larger, leading to a higher distribution-based MID at 6 months, making it a more time-dependant method than the anchor-based method.

A limitation of our study is that most of our included studies were noncontrolled. Another limitation of our study is that all our patients participated in trials investigating a BLVR treatment, which is known to be a highly effective intervention resulting in a large decrease in SGRQ scores after treatment [25]. This could affect the size of the MID estimates. Future studies, compromising multiple treatment modalities other than BLVR, are needed to confirm our findings.

A strength of our study is that we were able to maintain a high level of standardisation as all measurements were performed in one specialised research hospital in The Netherlands, applying the same settings/measurement sequence, using the same equipment. Furthermore, compared with most other MID determination studies, we had a relatively large sample size $[12,13,24]$.

In most studies determining a MID, results are solely expressed as absolute numbers. In our study, we have also determined the relative MIDs. Relative MIDs are able to evaluate changes adjusted for baseline SGRQ scores. We believe that when calculating a MID, besides the absolute MID, the relative MID should also be estimated.

In conclusion, this study is the first to establish a MID SGRQ specifically for patients with severe COPD. Using a combination of anchor- and distribution-based methods we established a MID 1 month after treatment of -8.3 units and a MID 6 months after treatment of -7.1 . The relative MID 1 month after treatment was $-13.4 \%$ and 6 months after treatment was $-11.1 \%$. Our new MID estimates could be applied for both interpreting SGRQ outcomes as well as sample size determination in future clinical trials investigating severe COPD patients.

\section{References}

1 Jones PW, Quirk FH, Baveystock CM. The St George's Respiratory Questionnaire. Respir Med 1991; 85: (Suppl. B), 25-31; discussion 33-37.

2 Copay AG, Subach BR, Glassman SD, et al. Understanding the minimum clinically important difference: a review of concepts and methods. Spine J 2007; 7: 541-546.

3 Jones PW. St. George's Respiratory Questionnaire: MCID. COPD 2005; 2: 75-79.

4 Slebos DJ, Klooster K, Ernst A, et al. Bronchoscopic lung volume reduction coil treatment of patients with severe heterogeneous emphysema. Chest 2012; 142: 574-582.

5 Klooster K, Ten Hacken NH, Slebos DJ. The lung volume reduction coil for the treatment of emphysema: a new therapy in development. Expert Rev Med Devices 2014; 11: 481-489.

6 Herth FJ, Eberhardt R, Gompelmann D, et al. Radiological and clinical outcomes of using Chartis to plan endobronchial valve treatment. Eur Respir J 2013; 41: 302-308.

7 Shah PL, Slebos DJ, Cardoso PF, et al. Bronchoscopic lung-volume reduction with Exhale Airway Stents for Emphysema (EASE trial): randomised, sham-controlled, multicentre trial. Lancet 2011; 378: 997-1005.

8 Deslee G, Klooster K, Hetzel M, et al. Lung volume reduction coil treatment for patients with severe emphysema: a European multicentre trial. Thorax 2014; 69: 980-986.

9 Klooster K, Ten Hacken NH, Franz I, et al. Lung volume reduction coil treatment in chronic obstructive pulmonary disease patients with homogeneous emphysema: a prospective feasibility trial. Respiration 2014; 88 : $116-125$

10 Donohue JF. Minimal clinically important differences in COPD lung function. COPD 2005; 2: 111-124.

11 Puhan MA, Chandra D, Mosenifar Z, et al. The minimal important difference of exercise tests in severe COPD. Eur Respir J 2011; 37: 784-790.

12 Hartman JE, Ten Hacken NH, Klooster K, et al. The minimal important difference for residual volume in patients with severe emphysema. Eur Respir J 2012; 40: 1137-1141.

13 Altenburg WA, Duiverman ML, Ten Hacken NH, et al. Changes in the endurance shuttle walk test in COPD patients with chronic respiratory failure after pulmonary rehabilitation: the minimal important difference obtained with anchor- and distribution-based method. Respir Res 2015; 16: 27.

14 Revicki D, Hays RD, Cella D, et al. Recommended methods for determining responsiveness and minimally important differences for patient-reported outcomes. J Clin Epidemiol 2008; 61: 102-109.

15 Jones PW, Quirk FH, Baveystock CM, et al. A self-complete measure of health status for chronic airflow limitation. The St. George's Respiratory Questionnaire. Am Rev Respir Dis 1992; 145: 1321-1327.

16 Miller MR, Hankinson J, Brusasco V, et al. Standardisation of spirometry. Eur Respir J 2005; 26: 319-338.

17 Wanger J, Clausen JL, Coates A, et al. Standardisation of the measurement of lung volumes. Eur Respir J 2005; 26: $511-522$. 
18 ATS Committee on Proficiency Standards for Clinical Pulmonary Function Laboratories. ATS statement: guidelines for the six-minute walk test. Am J Respir Crit Care Med 2002; 166: 111-117.

19 Calverley PM, Anderson JA, Celli B, et al. Salmeterol and fluticasone propionate and survival in chronic obstructive pulmonary disease. N Engl J Med 2007; 365: 775-789.

20 Tashkin DP, Celli B, Senn S, et al. A 4-year trial of tiotropium in chronic obstructive pulmonary disease. $N$ Engl J Med 2008; 359: 1543-1554.

21 Jones PW, Beeh KM, Chapman KR, et al. Minimal clinically important differences in pharmacological trials. Am J Respir Crit Care Med 2014; 189: 250-255.

22 Troosters T. How important is a minimal difference? Eur Respir J 2011; 37: 755-756.

23 Guyatt GH, Osoba D, Wu AW, et al. Methods to explain the clinical significance of health status measures. Mayo Clin Proc 2002; 77: 371-383.

24 Holland AE, Hill CJ, Rasekaba T, et al. Updating the minimal important difference for six-minute walk distance in patients with chronic obstructive pulmonary disease. Arch Phys Med Rehabil 2010; 91: 221-225.

25 Iftikhar IH, McGuire FR, Musani AI. Efficacy of bronchoscopic lung volume reduction: a meta-analysis. Int $J$ Chron Obstruct Pulmon Dis 2014; 9: 481-491. 